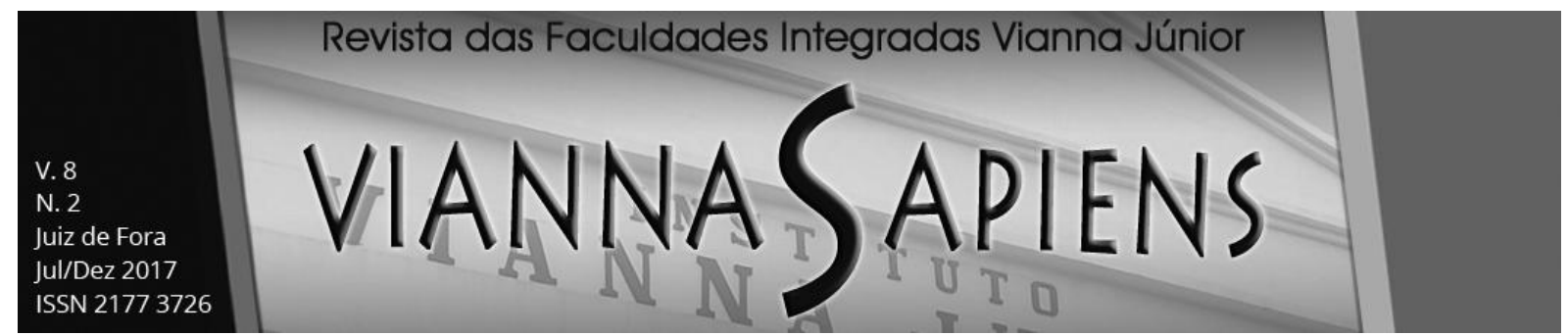

\title{
SATISFAÇÃO DOS USUÁRIOS DO METRÔ DO RIO DE JANEIRO:
}

um estudo realizado com modelagem de equações estruturais

\author{
Antônio Tomé R. M. dos Santos ${ }^{1}$ \\ Eduardo Dias Coutinho ${ }^{2}$ \\ Paulo Roberto da Costa Vieira ${ }^{3}$
}

\section{RESUMO}

A satisfação do cliente é de fundamental importância para garantir fluxo estável e crescente de consumidores, por meio da lealdade dos existentes e da comunicação oral positiva, atraindo novos clientes. O presente trabalho objetivou estudar a satisfação dos usuários de transporte público metroviário na cidade do Rio de Janeiro com os serviços prestados pelo MetrôRio. Na coleta de dados, empregou-se a pesquisa de survey, com utilização de questionário estruturado. Posteriormente, os dados foram tratados por meio da Modelagem de Equações Estruturais (MEE), com base em Mínimos Quadrados Parciais (MQP). Os resultados confirmaram que a satisfação do usuário sofre impacto estatisticamente significativo da qualidade do serviço, das expectativas e do valor percebido.

\section{PALAVRAS-CHAVE: SATISFAÇÃO DO CONSUMIDOR. MODELAGEM DE EQUAÇÕES ESTRUTURAIS. SERVIÇO DE METRÔ.}

\footnotetext{
${ }^{1}$ Mestrando em Administração e Desenvolvimento Empresarial (MADE/UNESA). Universidade Estácio de Sá (UNESA).e-mail: antoniotrms@gmail.com

2 Mestrando em Administração e Desenvolvimento Empresarial (MADE/UNESA). Universidade Estácio de Sá (UNESA), e-mail: ediascoutinho@yahoo.com.br

${ }^{3}$ Doutor em Administração (COPPEAD/UFRJ) - Professor Adjunto do Mestrado em Administração e Desenvolvimento Empresarial (MADE/UNESA). Universidade Estácio de Sá (UNESA). e-mail: paulo.vieira@estacio.br
} 


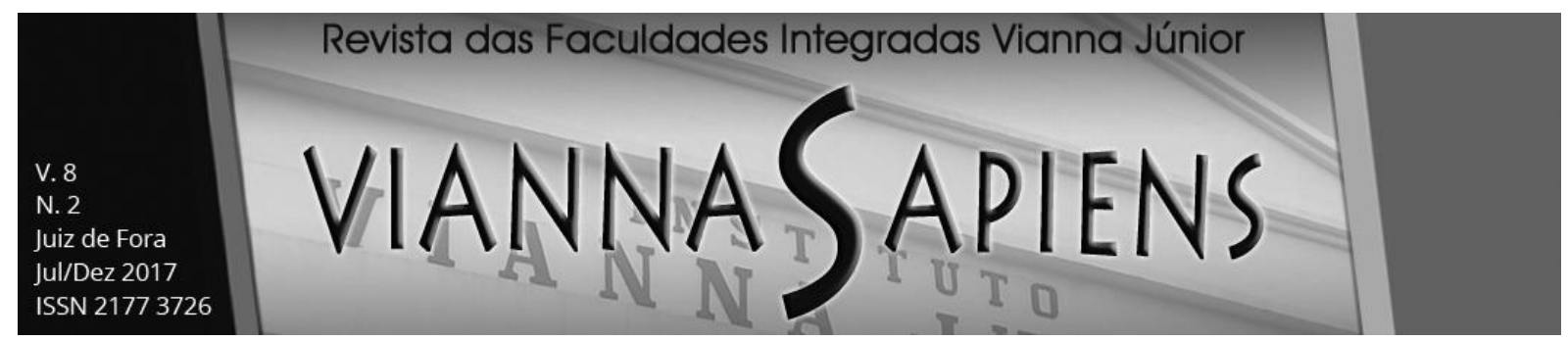

\section{INTRODUÇÃO}

O setor de serviços é caracterizado pela diversificação de ofertas aos consumidores e pela alta competitividade entre as empresas (MARCHETTI; PRADO, 2001). No Brasil, o desempenho das atividades do setor vem se destacando pela crescente participação na produção econômica (IBGE, 2017).

Há estudos que evidenciam que a satisfação é, em ambientes de competição acirrada, componente estratégico para retenção dos clientes e para ampliação da participação de mercado e da rentabilidade das empresas (FERNANDES; TEIXEIRA; LOPES, 2011).

A satisfação está relacionada à avaliação que o cliente faz sobre o serviço prestado pela empresa, com base em suas expectativas e percepções de qualidade (ZEITHAML; BITNER; GREMLER, 2014).

Apesar das empresas entenderem que a satisfação do consumidor é uma questão estratégica, a sua mensuração é tarefa complexa, devido às características inerentes ao serviço (LOVELOCK; WIRTZ; HEMZO, 2011).

Costa, Souza e Silva (2008) desenvolveram modelo para mensurar os fatores que determinam a satisfação dos consumidores da Companhia do Metropolitano de São Paulo - Metrô, responsável pelo transporte metroviário na cidade de São Paulo.

No presente estudo, utilizou-se o modelo desenvolvido por Costa, Souza e Silva (2008) para avaliar a satisfação dos consumidores da empresa Metrô Rio, responsável pelo serviço de transporte metroviário na cidade do Rio de Janeiro.

\section{REFERENCIAL TEÓRICO}

A satisfação dos consumidores se tornou um dos principais temas na literatura de marketing de serviços. Diferentes estudos evidenciam a contribuição da satisfação dos clientes para obtenção de melhores resultados corporativos (FERNANDES; TEIXEIRA; LOPES, 2011). 


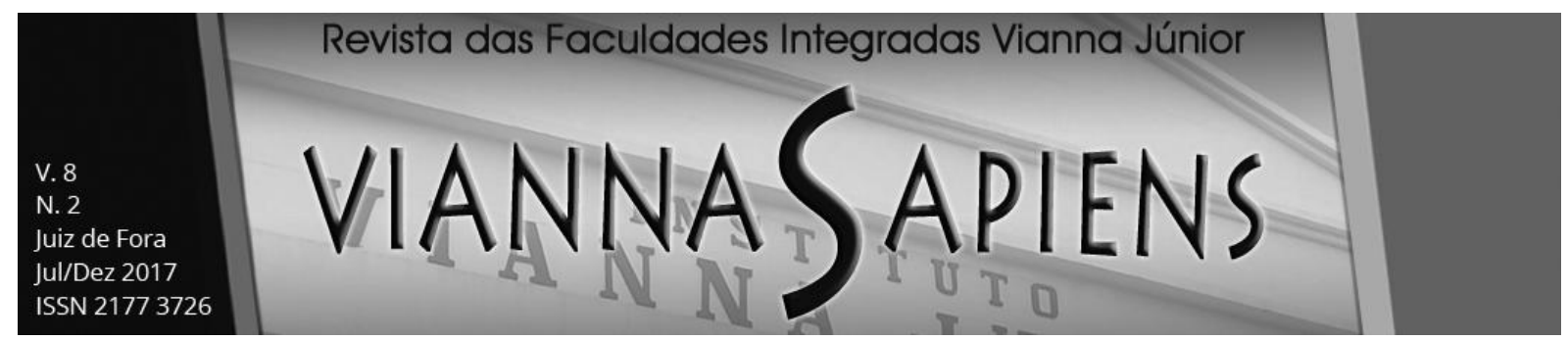

$\mathrm{Na}$ literatura de marketing de serviços existem diversos modelos habilitados a mensurar a satisfação dos consumidores. Devido à diversificação de ofertas existentes no setor de serviços, os modelos são construídos, considerando as particularidades de cada situação.

No presente trabalho, utilizou-se o modelo desenvolvido por Costa, Souza e Silva (2008) para analisar a satisfação dos consumidores de serviço de transporte público metroviário prestado pela empresa MetrôRio. Esse modelo supõe a existência de nexo causal entre os construtos exógenos (Qualidade Percebida; Expectativas dos Consumidores; e Valor Percebido) e o endógeno (Satisfação do Consumidor) (Figura 1).

O modelo utilizado neste trabalho atua da seguinte forma: 1) a expectativa do consumidor quanto à qualidade do serviço que será prestado influencia a qualidade percebida (primeira hipótese); 2) a qualidade percebida exerce efeito direto e positivo sobre a satisfação do consumidor (segunda hipótese); 3) a qualidade percebida exerce efeito direto e positivo sobre o valor percebido (terceira hipótese); 4) a aludida expectativa do consumidor exerce efeito direto e positivo sobre o valor percebido (quarta hipótese); 5) o valor percebido exerce efeito direto e positivo sobre a satisfação do consumidor (quinta hipótese); e 6) a expectativa em tela exerce efeito direto e positivo sobre a satisfação do consumidor (sexta hipótese) (COSTA; SOUZA; SILVA, 2008). 

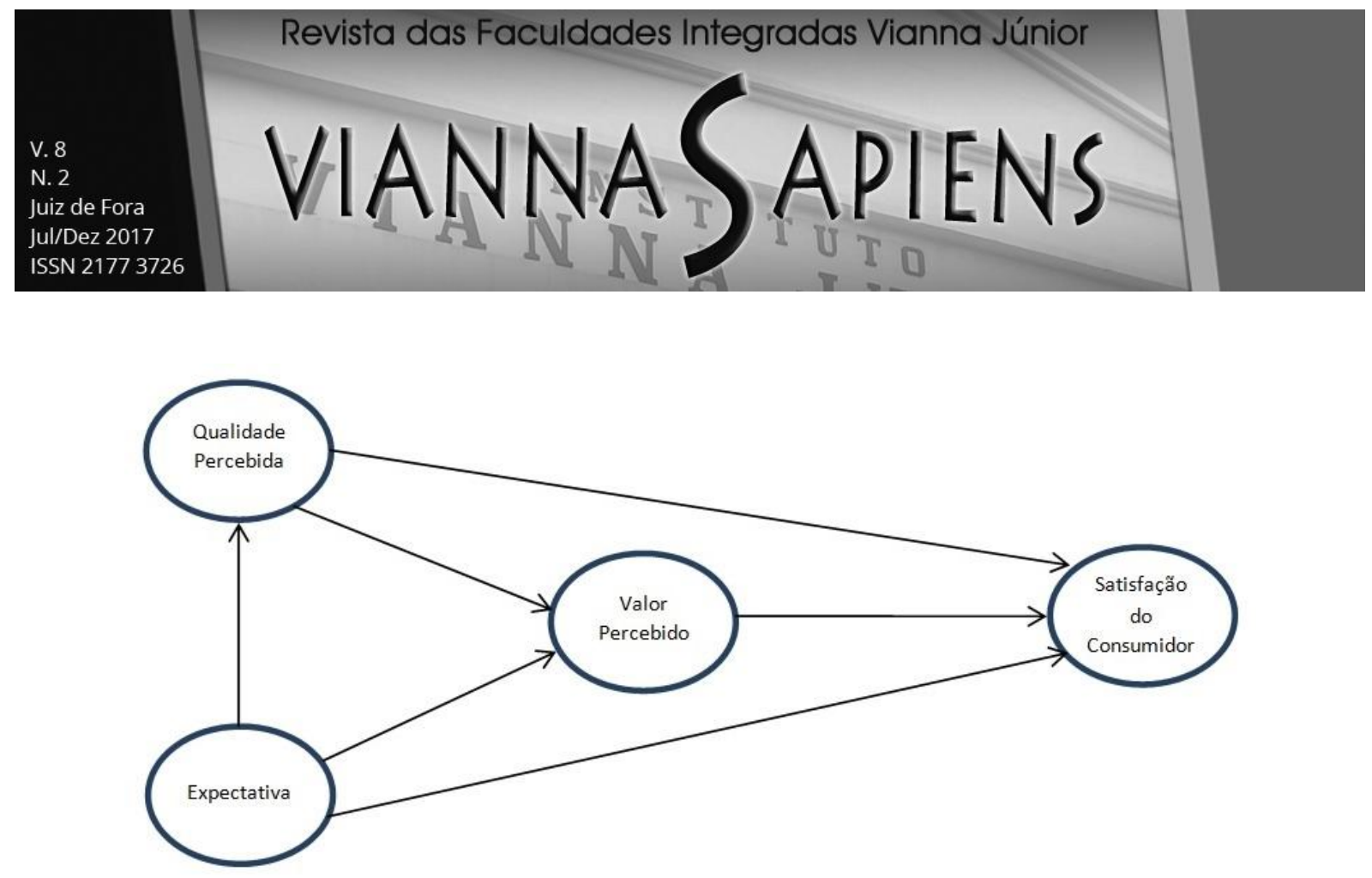

Figura 1 - Modelo Costa, Souza e Silva (2008)

Fonte: Costa, Souza e Silva (2008).

As expectativas do consumidor, no que diz respeito à qualidade do serviço, exercem efeito direto e positivo em sua satisfação (FORNELL et al., 1996). As expectativas estão relacionadas às previsões que o cliente faz sobre a qualidade do serviço que the será oferecido (ANDERSON; FORNELL; LEHMANN, 1994; GRÖNROOS, 1984; SPRENG; MACKENZIE; OLSHAVSKY, 1996) Por meio de expectativas, o cliente cria padrões de qualidade que norteiam sua avaliação na experiência de consumo (OLIVER, 1980; Yi, 1993).

Antes de adquirir o produto ou serviço, o consumidor cria expectativas por meio de diversas fontes, como publicidade, precificação, experiências anteriores, notícias na mídia, comunicação boca a boca e valores intrínsecos às pessoas (ANDERSON; FORNELL; LEHMAN, 1994).

Essas fontes de construção das expectativas são formadas por componentes baseados no passado e no futuro. As experiências e as informações obtidas pelos consumidores no passado servem de base para a construção das expectativas de consumo no futuro. Essas expectativas influenciam a qualidade percebida pelos clientes e a sua satisfação (ANDERSON; FORNELL; LEHMAN, 1994). 


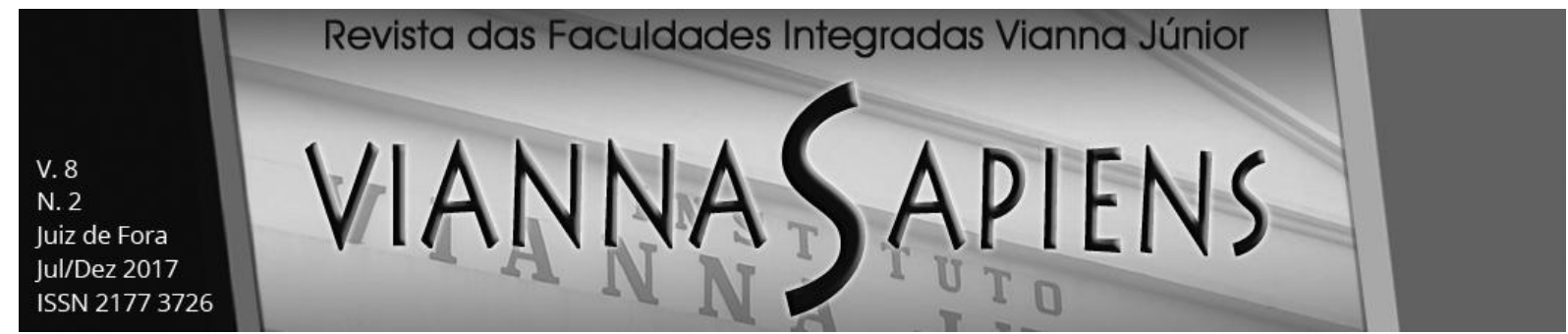

A qualidade percebida, um dos determinantes da satisfação do consumidor, refere-se à avaliação do cliente sobre a superioridade ou a excelência global do serviço (PARASURAMAN; ZEITHAML; BERRY, 1988; ZEITHAML, 1988).

A avaliação da qualidade se baseia, principalmente, em dois componentes inerentes à experiência de consumo. O primeiro diz respeito à customização, nível em que a empresa personaliza seus produtos ou serviços para atender às necessidades heterogêneas dos clientes. O segundo se refere à confiabilidade, grau em que os produtos ou serviços são confiáveis, padronizados e livres de defeitos (FORNELL et al., 1996).

Outro determinante da satisfação é o valor percebido pelo consumidor (FORNELL et al., 1996; ANDERSON; FORNELL, 2000). Refere-se à comparação que o cliente faz sobre os benefícios e os custos relativos ao serviço fornecido pela empresa (ZEITHAML; BITNER; GREMLER, 2014), também explicado como a percepção de todos os atributos da qualidade confrontados com o preço do serviço (WOODRUFF, 1997; ANDREASSEN; LINDESTAD, 1998).

A satisfação dos consumidores, variável exógena do modelo utilizado neste trabalho, recebe a influência de todas as outras variáveis (FORNELL et al., 1996). Trata-se do julgamento posterior à avaliação de determinada compra (CHURCHILL; SURPRENANT, 1982; BEARDEN; TEEL, 1983; OLIVER; DESARBO, 1988).

O conceito da satisfação tem íntima relação com 0 atendimento das expectativas dos clientes, assim como com suas percepções de qualidade e de valor (FORNELL, 1992; FORNELL et al, 1996). Portanto, o julgamento da satisfação é inteiramente subjetivo e individual (OLIVER, 1999).

As expectativas dos consumidores sobre a qualidade do serviço influenciam diretamente suas satisfações. Caso a empresa forneça serviço com qualidade superior às expectativas dos clientes, haverá desconformidade positiva, resultando na satisfação do consumidor, por outro lado, se a percepção de qualidade for inferior às expectativas, haverá desconformidade negativa, o que poderá resultar na insatisfação do cliente (OLIVER, 1980; PARASURAMAN; ZEITHAML; BERRY, 1994). 


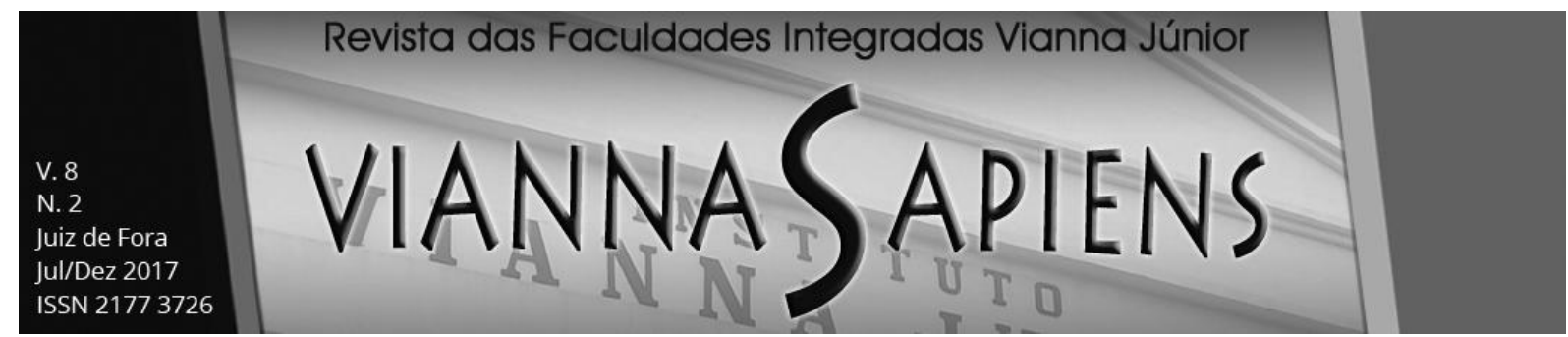

A satisfação é indicador estratégico às corporações, já que influencia positivamente à retenção dos clientes, contribuindo para geração de fluxos de caixa estáveis e crescentes, dado que clientes satisfeitos tecem comentários positivos acerca da oferta da firma (SÁNCHEZ-GARCíA et al, 2012).

\section{METODOLOGIA}

A presente pesquisa é de natureza quantitativa, com enfoque explicativo, já que considera relações causais entre as variáveis latentes do modelo. Com o objetivo de obter um número expressivo de informações por respondente, empregou-se a pesquisa de survey.

\subsection{População e Amostra}

A população desta pesquisa é composta por todos os usuários de serviço de transporte prestado pela MetrôRio.

A amostra foi do tipo não-probabilística, escolhida por conveniência, composta por 235 usuários do serviço prestado pela empresa MetrôRio.

\subsection{Coleta de dados}

Os dados foram coletados por meio de questionário estruturado e autoadministrado, com escala Likert de cinco opções de resposta, baseado no modelo desenvolvido por Costa, Souza e Silva (2008). Cada item do questionário corresponde a um indicador (variável observada) do modelo de mensuração associado ao modelo hipotético. A Tabela 1 exibe esses indicadores (variáveis observadas), os quais foram agrupados por variáveis latentes (construtos) do modelo hipotético de satisfação do usuário de metrô. 


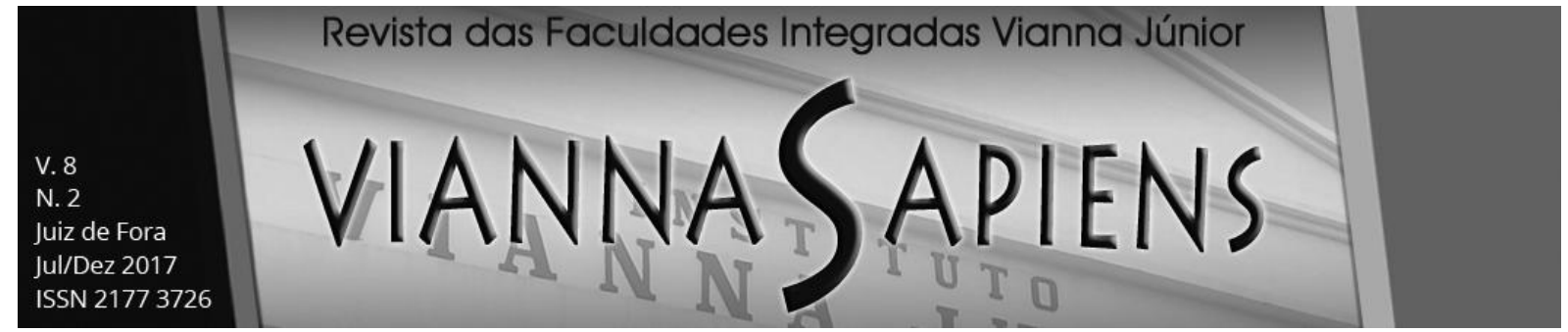

Tabela 1 - Indicadores e variáveis latentes do modelo de Costa, Souza e Silva INDICADORES VARIÁVEIS LATENTES

1. Expectativa geral em relação à qualidade

2. Expectativa em relação à customização

Expectativa

3. Expectativa em relação à confiabilidade

4. Avaliação geral sobre a qualidade experimentada

5. Avaliação da customização experimentada

Qualidade Percebida

6. Avaliação da confiabilidade experimentada

7. Avaliação da qualidade, dado o preço

8. Avaliação do preço, dada a qualidade

Valor Percebido

9. Satisfação geral com o serviço

10. Desconfirmação das expectativas Satisfação do Consumidor

11. Desempenho versus concepção de serviço ideal

Fonte: Costa, Souza e Silva (2008).

\subsection{Tratamento dos Dados}

No presente trabalho, foi utilizado o software WarpPLS, versão 5.0, para tratar os dados por meio de modelagem de equações estruturais (MEE), com base em mínimos quadrados parciais (MQP), que foi escolhida devido à complexidade do modelo, ao tamanho da amostra e ao desconhecimento da normalidade das variáveis.

\section{RESULTADOS}

A análise de resultados desta pesquisa foi realizada em duas etapas. A primeira etapa foi responsável por avaliar o modelo de mensuração. A segunda etapa teve o objetivo de avaliar o modelo estrutural. Trata-se do procedimento mais 


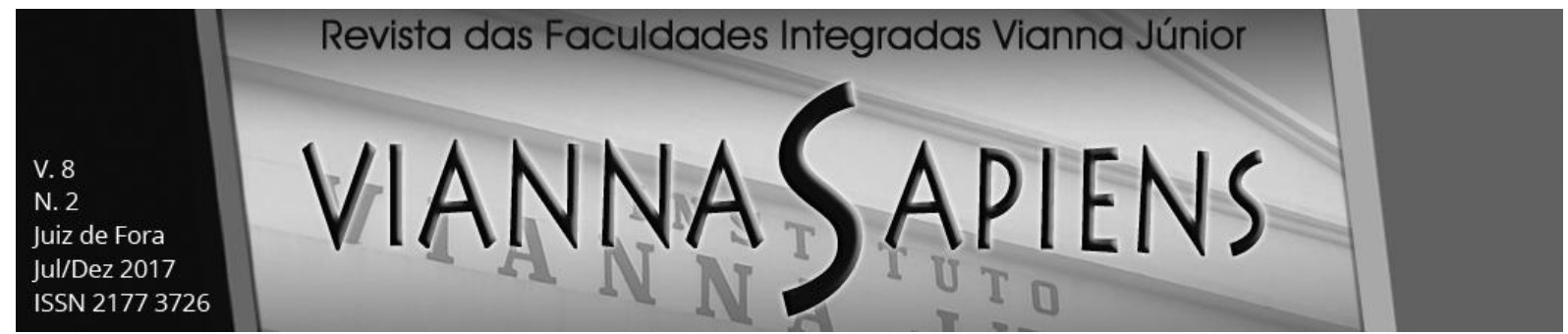

indicado quando os dados são tratados por meio de MEE, com MQP (HENSELER; RINGLE; SINKOVICS, 2009; HAIR et al., 2014a).

O modelo de mensuração, também referenciado como modelo externo, exibe as relações entre as variáveis latentes e as variáveis observadas. A sua avaliação, em modelos reflexivos, ocorre por meio da análise de confiabilidade composta (CC), de variância média extraída (VME), e de cargas cruzadas, sendo, complementarmente, utilizado o critério de Fornell e Larcker (1981) (HENSELER; RINGLE; SINKOVICS, 2009; RINGLE; SILVA; BIDO, 2014; HAIR et al., 2014a; HAIR et al., 2014b).

A análise de confiabilidade composta (CC) é responsável por mensurar a confiabilidade da consistência interna dos indicadores. Ela avalia com precisão adequada se a amostra não tem vieses e se os indicadores são capazes de extrair informações confiáveis. Entende-se que valores compreendidos entre 0,70 a 0,90 são satisfatórios (HAIR et al., 2014a; HAIR et al., 2014b; RINGLE; SILVA; BIDO, 2014).

Apesar do alfa de Cronbach não ser um indicador indispensável em MEE, com MQP, ele foi analisado no presente trabalho. Espera-se que o alfa de Cronbach esteja acima de 0,70, não obstante sejam aceitáveis valores superiores a 0,60, quando se trata de pesquisa em Ciências Sociais (HAIR et al., 2014b).

No que se refere à análise da variância média extraída (VME), o objetivo se concentra na verificação da validade convergente, que pode ser explicada pela extensão em que uma medida se correlaciona de forma positiva com medidas alternativas do mesmo construto. O valor da VME de cada variável latente precisa ser igual ou superior a 0,50 , pois isso demonstra que, em média, o construto explica mais do que a média de variância de seus indicadores (HAIR et al., 2014a; HAIR et al., 2014b; HENSELER; HUBONA; RAY, 2016).

No presente estudo, conforme pode ser visualizado na Tabela 2, os valores encontrados na análise de CC e de VME são adequados. O alpha de Cronbach da variável latente Satisfação do Consumidor (SATI) apresentou valor entre 0,60 e 0,70. 


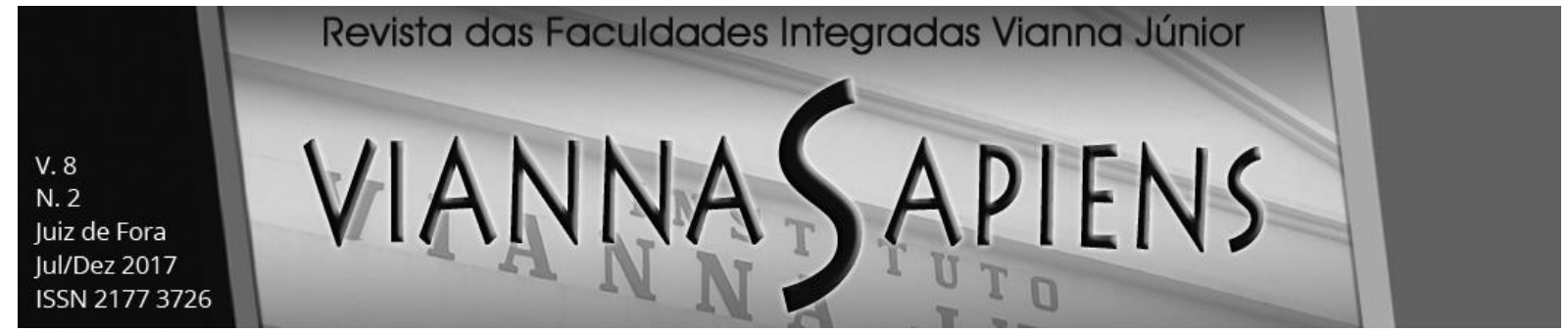

Tabela 2 - Alpha de Cronbach, Confiabilidade Composta (CC) e Variância Média Extraída (VME)

\begin{tabular}{cccc}
\hline Variável Latente & $\begin{array}{c}\text { Alpha de } \\
\text { Cronbach }\end{array}$ & CC & VME \\
\hline Expectativa (EXPE) & 0,872 & 0,922 & 0,797 \\
Qualidade Percebida (QPER) & 0,826 & 0,896 & 0,742 \\
Valor Percebido (VPER) & 0,895 & 0,950 & 0,905 \\
Satisfação do Consumidor (SATI) & 0,612 & 0,796 & 0,576 \\
\hline
\end{tabular}

Fonte: Elaboração própria.

No que diz respeito à avaliação das cargas cruzadas ou ao critério de Fornell e Larcker (1981), o intuito é analisar a validade discriminante dos construtos, ou seja, o quanto eles são independentes um dos outros (HAIR et al., 2014b).

O exame das cargas cruzadas tem o objetivo de analisar se os indicadores possuem cargas fatoriais mais elevadas em seus respectivos construtos. O critério de Fornell e Lacker (1981) é uma abordagem mais conservadora para avaliar a validade discriminante, pois verifica se a raiz quadrada de cada VME é superior a sua maior correlação com qualquer outra variável latente do modelo (HAIR et al., 2014b; HENSELER; HUBONA; RAY, 2016).

No presente trabalho, os resultados obtidos atendem aos dois critérios mencionados para avaliar a validade discriminante dos construtos, conforme pode ser observado nas Tabelas 3 e 4. 


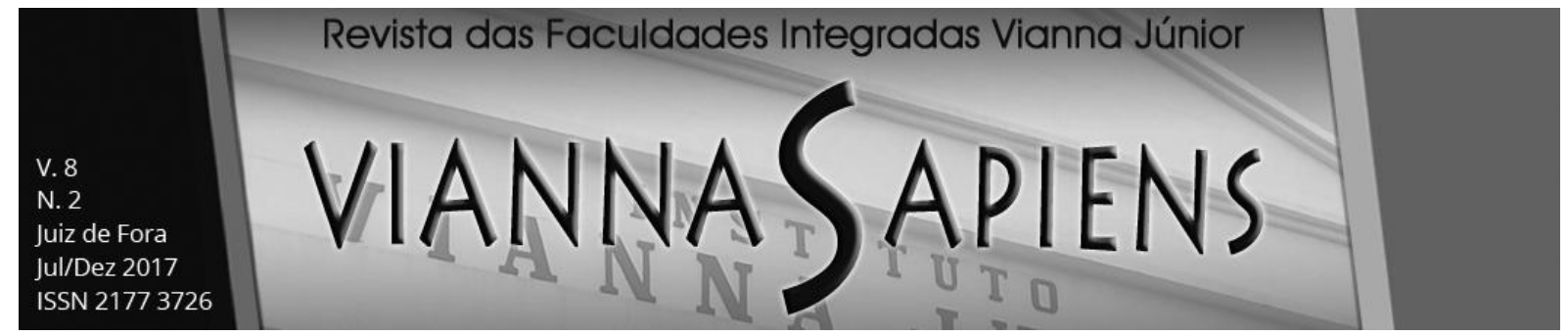

Tabela 3-Avaliação das Cargas Cruzadas

\begin{tabular}{lcccc}
\hline & EXPE & QPER & VPER & SATI \\
\hline EXPE1 & $\mathbf{( 0 . 9 1 5 )}$ & 0.071 & 0.053 & -0.007 \\
EXPE2 & $\mathbf{( 0 . 8 9 8 )}$ & -0.195 & 0.019 & 0.102 \\
EXPE3 & $\mathbf{( 0 . 8 6 4 )}$ & 0.128 & -0.075 & -0.099 \\
QPER1 & 0.169 & $\mathbf{( 0 . 8 7 3 )}$ & 0.134 & 0.063 \\
QPER2 & -0.170 & $\mathbf{( 0 . 8 5 6 )}$ & -0.030 & 0.040 \\
QPER3 & -0.002 & $\mathbf{( 0 . 8 5 5 )}$ & -0.107 & -0.105 \\
VPER1 & 0.069 & -0.090 & $\mathbf{( 0 . 9 5 1 )}$ & -0.081 \\
VPER2 & -0.069 & 0.090 & $\mathbf{( 0 . 9 5 1 )}$ & 0.081 \\
SATI1 & 0.005 & 0.367 & 0.217 & $\mathbf{( 0 . 8 3 7 )}$ \\
SATI2 & 0.014 & -0.176 & 0.009 & $\mathbf{( 0 . 8 7 0 )}$ \\
SATI3 & -0.032 & -0.296 & -0.364 & $\mathbf{( 0 . 5 2 0 )}$ \\
\hline
\end{tabular}

Fonte: Elaboração própria.

Tabela 4-Critério de Fornell-Larcker

\begin{tabular}{lllll}
\hline & EXPE & QPER & VPER & SATI \\
\hline EXPE & $\mathbf{0 . 8 9 3}$ & & & \\
QPER & 0.738 & $\mathbf{0 . 8 6 1}$ & & \\
VPER & 0.469 & 0.605 & $\mathbf{0 . 9 5 1}$ & \\
SATI & 0.601 & 0.734 & 0.552 & $\mathbf{0 . 7 5 9}$ \\
\hline
\end{tabular}

Fonte: Elaboração própria.

Antes de iniciar a análise do modelo estrutural, é recomendável verificar o fator de inflação de variância (VIF) para identificar possíveis problemas de multicolinearidade entre as variáveis latentes. Construtos com valores de VIF abaixo de 5 são satisfatórios, possibilitando rejeitar a hipótese de multicolinearidade entre os construtos do modelo (HAIR et al., 2014b). Conforme pode ser observado na tabela 5 , não há problemas de multicolinearidade entre as variáveis latentes. 


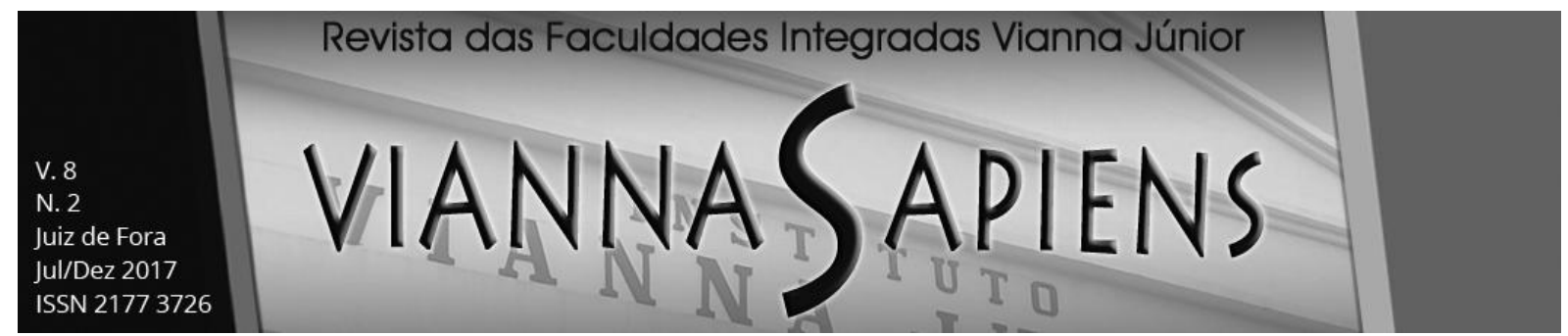

Tabela 5 - Fator de Inflação de Variância (VIF)

\begin{tabular}{cc}
\hline Variável Latente & VIF \\
\hline Expectativa & 2.237 \\
Qualidade Percebida & 3.388 \\
Valor Percebido & 1.644 \\
Satisfação do Consumidor & 2.290 \\
\hline
\end{tabular}

Fonte: Elaboração própria.

O modelo estrutural desta pesquisa foi analisado por meio da verificação do coeficiente de determinação de Pearson $\left(R^{2}\right)$, da avaliação da significância estatística dos coeficientes de caminho $(\beta)$, da avaliação da relevância preditiva $\left(Q^{2}\right)$ e da avaliação do tamanho do efeito $\left(\mathfrak{f}^{2}\right)$, por serem as medidas mais adequadas para avaliar o modelo interno em MEE, com MQP (HAIR et al., 2014b).

O coeficiente de determinação de Pearson $\left(R^{2}\right)$ é a medida mais utilizada para avaliar o modelo estrutural em MEE, com MQP. Trata-se de uma medida preditiva do modelo. $\mathrm{O} \mathrm{R}^{2}$ analisa os efeitos das variáveis latentes exógenas sobre as variáveis latentes endógenas. Os valores de $R^{2}$ variam de 0 a 1 . Quanto mais os valores se aproximam de 1, maior é a exatidão preditiva (HAIR et al., 2014b).

Como é possível verificar na Figura 2, a Satisfação do Consumidor, principal variável latente endógena do modelo, apresentou valor $R^{2}$ de 0,567 . As variáveis latentes Qualidade Percebida e Valor Percebido são exógenas e endógenas ao mesmo tempo. Elas apresentaram valores $\mathrm{R}^{2}$ de 0,547 e 0,374, respectivamente. 

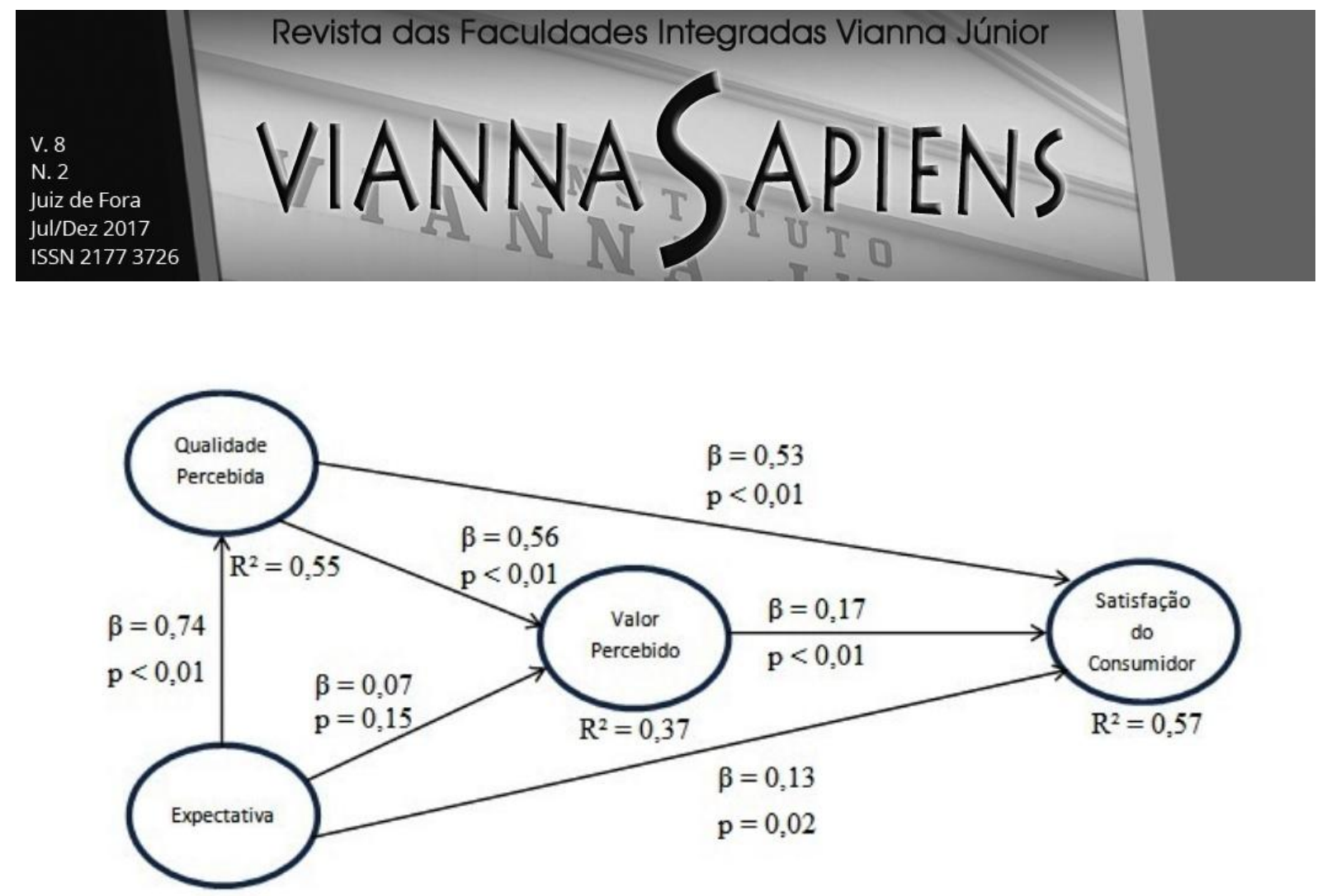

Figura 2 - Diagrama de Caminho

Fonte: Elaboração própria

No que se refere à avaliação da significância estatística dos coeficientes de caminho ( $\beta$ ), é importante ressaltar que o $\beta$ representa as relações hipotéticas entre os construtos, que só podem ser aceitas se houver significância estatística. Os resultados dos coeficientes sempre estão contidos entre -1 e 1, variando de uma relação negativa à positiva, sendo que quanto mais próximo os valores estiverem dos extremos, mais forte serão as relações entre os construtos (HAIR et al., 2014b).

$O$ coeficiente de caminho entre os construtos Expectativa e Valor Percebido não exibiu significância estatística $(p=0,146)$. As demais relações entre os construtos do modelo utilizados neste trabalho tiveram significância estatística, conforme demonstrado na Figura 2 e na Tabela 6. 


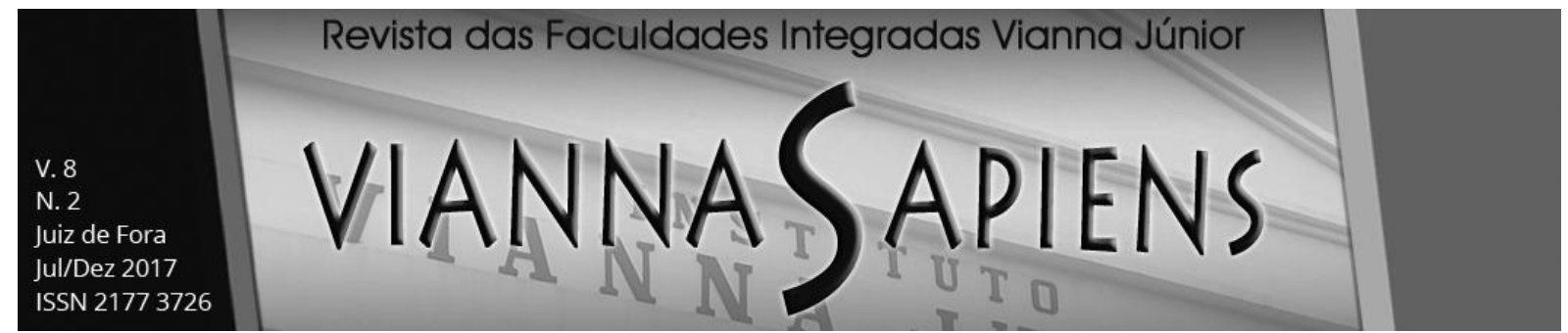

Tabela 6 - Coeficientes de caminho $(\beta)$ e significância estatística $(p)$

\begin{tabular}{ccc}
\hline Caminho & B & P \\
\hline EXPE $\rightarrow$ QPER & 0,740 & $<0,001$ \\
EXPE $\rightarrow$ VPER & $\mathbf{0 , 0 6 8}$ & $\mathbf{0 , 1 4 6}$ \\
EXPE $\rightarrow$ SATI & 0,134 & 0,018 \\
QPER $\rightarrow$ VPER & 0,561 & $<0,001$ \\
QPER $\rightarrow$ SATI & 0,534 & $<0,001$ \\
VPER $\rightarrow$ SATI & 0,170 & 0,004 \\
\hline
\end{tabular}

Fonte: Elaboração própria.

Sobre a avaliação da relevância preditiva $\left(Q^{2}\right)$, também conhecido como indicador de Stone-Geisser, cabe mencionar que o objetivo é analisar quanto o modelo se aproxima do que se espera dele. Valores de 0,02;0,15; e 0,35 indicam que um construto exógeno tem relevância preditiva pequena, média ou grande (RINGLE; SILVA; BIDO, 2014; HAIR et al., 2014b).

Os resultados encontrados neste trabalho indicaram que a principal variável endógena do modelo, Satisfação do consumidor, teve o $Q^{2}$ de 0,570. As outras duas variáveis latentes do modelo, Qualidade Percebida e Valor Percebido apresentaram valores $Q^{2}$ de 0,547 e 0,374, respectivamente.

Por último, no que se refere à avaliação do tamanho do efeito $\left(f^{2}\right)$, também conhecido como indicador de Cohen (1988), é importante ressaltar que os valores 0,02, 015 e 0,35, representam efeitos pequeno, médio e grande da variável latente exógena, respectivamente (HAIR et al., 2014b).

Os resultados encontrados no presente trabalho podem ser observados na tabela 7. 


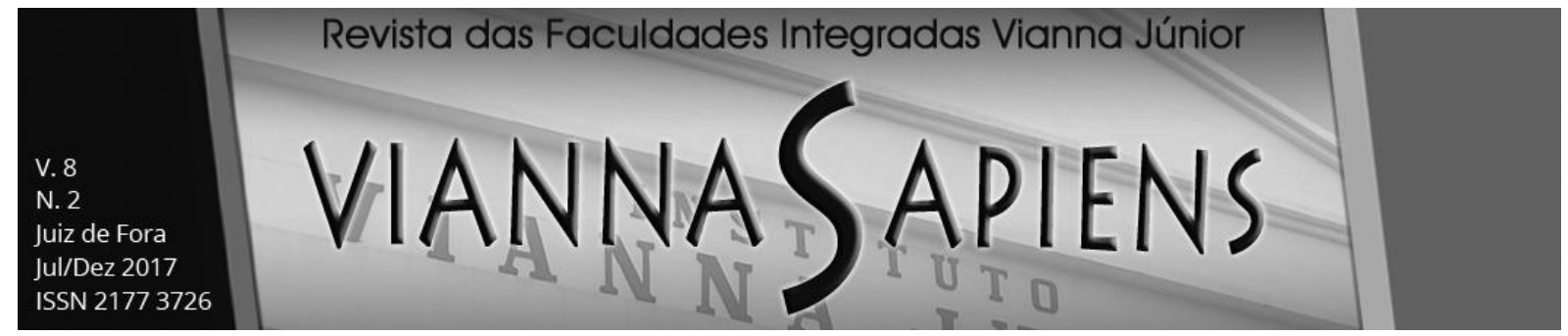

Tabela 7 - Tamanho do efeito $\left(\mathbf{f}^{2}\right)$

\begin{tabular}{cc}
\hline Caminho & $\mathbf{f}^{\mathbf{2}}$ \\
\hline EXPE $\rightarrow$ QPER & 0,547 \\
EXPE $\rightarrow$ VPER & 0,032 \\
EXPE $\rightarrow$ SATI & 0,081 \\
QPER $\rightarrow$ VPER & 0,342 \\
QPER $\rightarrow$ SATI & 0,392 \\
VPER $\rightarrow$ SATI & 0,094 \\
\hline
\end{tabular}

Fonte: Elaboração própria.

Os resultados encontrados não suportaram a hipótese de a Expectativa exercer efeito direto e positivo sobre o Valor Percebido, conforme pode ser visualizado na Tabela 8.

Tabela 8 - Confirmação ou rejeição das hipóteses fundamentais do modelo hipotético de satisfação

\begin{tabular}{lc}
\hline \multicolumn{1}{c}{ Hipótese } & Resultado obtido \\
\hline H1:Expectativa exerce efeito direto e positivo sobre a & Confirma H1 \\
Qualidade Percebida. & Confirma H2 \\
H2: Qualidade Percebida exerce efeito direto e positivo & Confirma H3 \\
sobre a Satisfação do Consumidor. & Rejeita H4 \\
H3: Qualidade Percebida exerce efeito direto e positivo & sobre o Valor Percebido. \\
H4: Expectativa exerce efeito direto e positivo sobre o & Confirma H5 \\
Valor Percebido. & H5: Valor Percebido exerce efeito direto e positivo sobre \\
a Satisfação do Consumidor. \\
H6: Expectativa exerce efeito direto e positivo sobre a \\
Satisfação do Consumidor.
\end{tabular}

Fonte: Elaboração própria. 


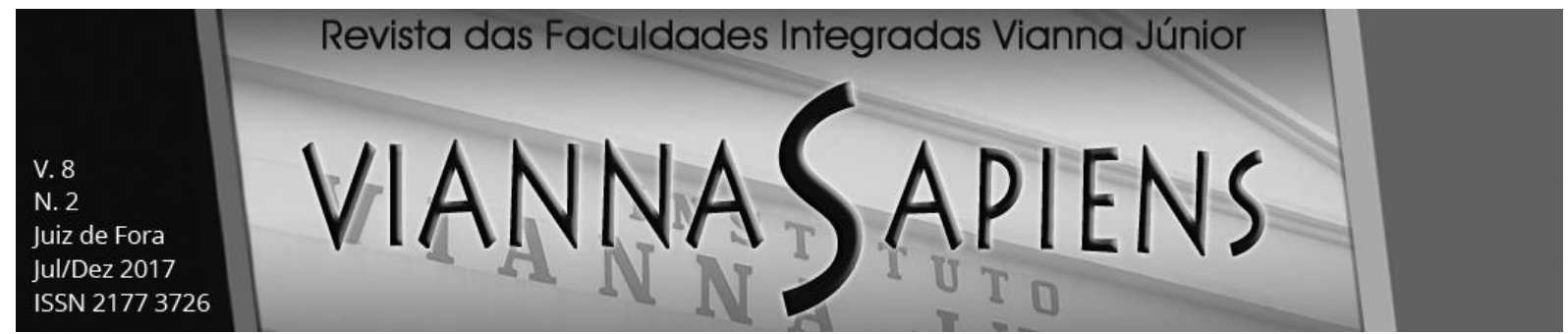

CONCLUSÃO

O presente estudo aplicou o modelo desenvolvido por Costa, Souza e Silva (2008) na empresa MetrôRio, com o objetivo de investigar a consistência dos elos causais entre os construtos do modelo, extraindo insights que possam ampliar a satisfação do cliente.

Os resultados apontam que há influência direta e positiva das variáveis Expectativa, Valor Percebido e Qualidade Percebida na Satisfação do Consumidor, principal variável endógena do modelo, sendo que a Qualidade Percebida foi a variável exógena que mais influenciou a Satisfação do Consumidor, corroborando com os resultados encontrados por Costa, Souza e Silva (2008) e com a literatura de marketing de serviços, que reconhece a importância da qualidade percebida para as satisfações dos consumidores.

Os resultados deste trabalho ratificam os estudos de Fornell (1992), onde a variável Qualidade Percebida também evidenciou a maior influência na Satisfação dos Consumidores.

É importante ressaltar que os resultados também identificaram que as expectativas dos consumidores influenciam significativamente suas percepções de qualidade, indo ao encontro de resultados empíricos de diversos autores.

Em contrapartida, os resultados encontrados não suportam a hipótese da expectativa como antecedente direto do valor percebido. É importante mencionar que na pesquisa de Costa, Souza e Silva (2008), esta relação hipotética também não foi confirmada diretamente.

Os serviços prestados pelo MetrôRio são, geralmente, de boa qualidade, o que implica dizer que a expectativa quanto à prestação adequada de serviço vai ao encontro da verdadeira qualidade. A expectativa não interfere diretamente no valor, mas somente por meio da qualidade percebida, já que a expectativa de qualidade e a qualidade percebida são sobrepostas. 


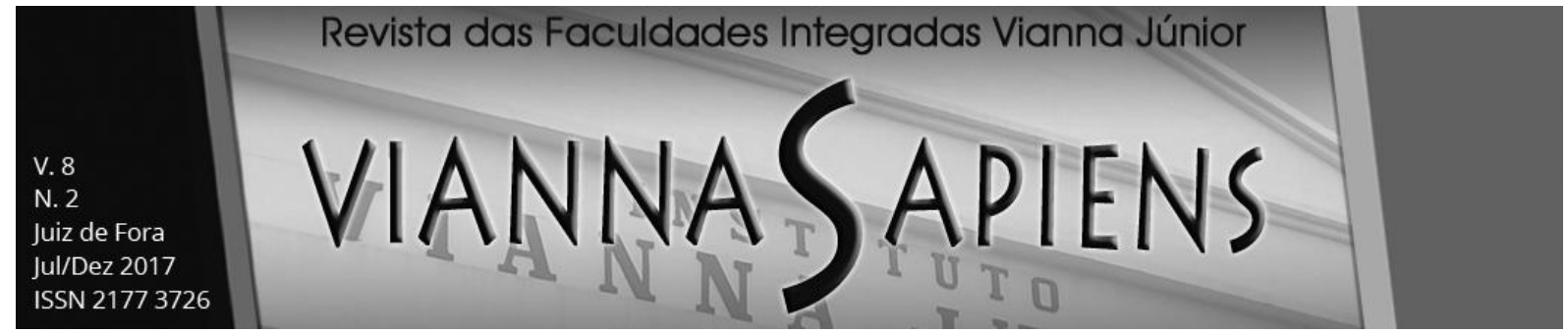

Com efeito, a qualidade do serviço é fundamental, uma vez que ela é a variável latente que maior impacto direto exerce sobre a satisfação, além de exercer impacto indireto via valor percebido.

Segue-se, portanto, que, para manter os clientes satisfeitos, o MetrôRio não pode descuidar da qualidade do serviço, zelando diuturnamente para que os horários e intervalos entre composições sejam rigorosamente observados; a limpeza dos vagões seja primorosa; e a segurança em seu interior seja garantida aos clientes, com resposta rápida e efetiva a qualquer situação anormal.

Por fim, observou-se, que os clientes da MetrôRio valorizam o atendimento de suas necessidades individuais e da confiabilidade do serviço, principalmente, no que se refere à ausência de falhas e à uniformidade do atendimento.

\title{
SATISFACTION OF METRO USERS OF RIO DE JANEIRO: A study with Structural Equations Modeling
}

\begin{abstract}
Customer satisfaction is of fundamental importance to ensure steady and growing flow of consumers by loyalty of existing clients and from its positive oral communication, attracting new customers. The present study aimed to study the satisfaction of users of public transportation in the city of Rio de Janeiro with the services rendered by MetrôRio. In the data collection, a survey has been employed, using the structured questionnaire. Subsequently, the data has been processed by Structural Equations Modeling (SEM), it based on Partial Least Squares (PLS). The results confirmed that user satisfaction has a statistically significant impact on the quality of the service, on the expectations and on the perceived value.
\end{abstract}




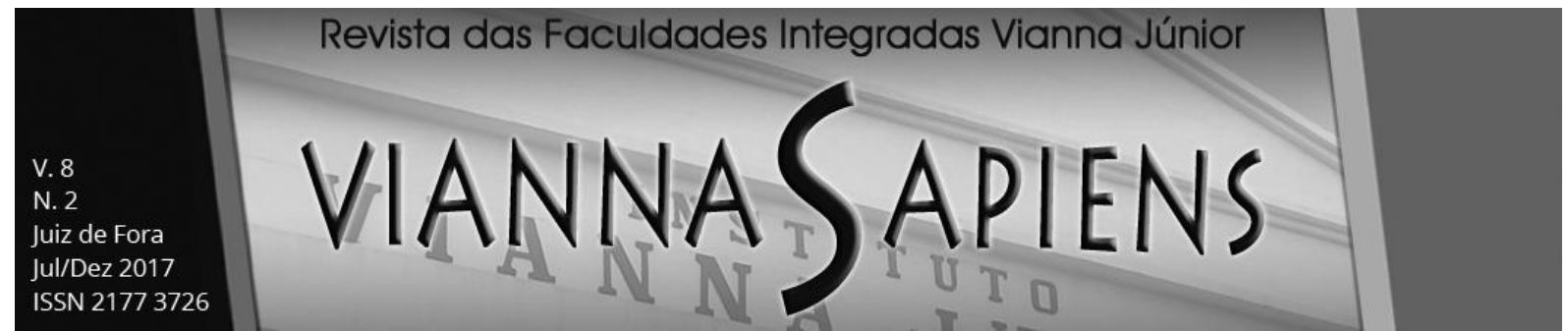

KEY WORDS: CONSUMER SATISFACTION. STRUCTURAL EQUATIONS MODELING. METRO SERVICE.

\section{REFERÊNCIAS}

ANDERSON, E. W.; FORNELL, C.; LEHMANN, D. R. Customer Satisfaction, Market Share, and Profitability: findings from Sweden. Journal of Marketing, v. 58, n. 3, p. 53-66, 1994.

ANDERSON, E. W.; FORNELL, C. Foundations of the American Customer Satisfaction Index. Total Quality Management, v. 11, n. 7, p. 869-882, 2000.

ANDREASSEN, T. W.; LINDESTAD, B. Customer loyalty and complex services The impact of corporate image on quality, customer satisfaction and loyalty for customers with varying degrees of service expertise. International Journal of Service Industry Management, v. 9, n. 1, p. 7-23, 1998.

BEARDEN, W. O.; TEEL, J. E. Selected Determinants of Consumer Satisfaction and Complaint Reports. Journal of Marketing Research, v. 20, p. 21-28, 1983.

COSTA, A. C. F.; SOUZA, S. S.; SILVA, L. C. T. Investigação sobre a satisfação do usuário dos serviços prestados pelo Metrô de São Paulo: um estudo exploratório, descritivo e ilustrativo com a utilização do modelo de equações estruturais. Revista de Gestão da USP, v. 15, n. especial, p. 93-108, 2008.

CHURCHILL, G. A.; SURPRENANT, C. An Investigation into the Determinants of Customer Satisfaction. Journal of Marketing Research, v. 19, p. 491-504, 1982.

COHEN, J. Statistical Power Analysis for the Behavioral Sciences. 2 ed. New York: Psychology Press, 1988. 


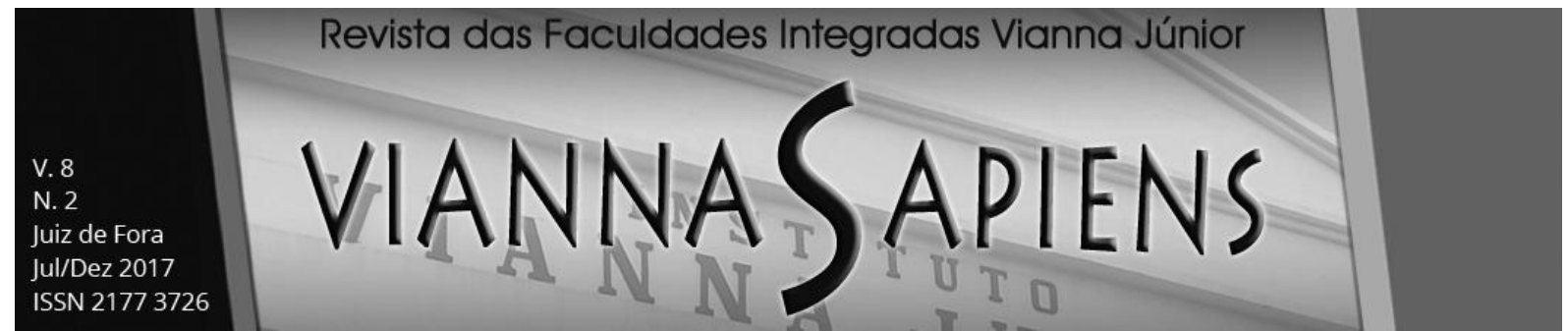

FERNANDES, J. C. S. O.; TEIXEIRA, D. J.; LOPES, H. E. G. A satisfação do cliente como fator estratégico em uma escola de idiomas. Revista Economia \& Gestão, v. 11, n. 26, p. 110-128, 2011.

FORNELL, C. A National Customer Satisfaction Barometer: The Swedish Experience. Journal of Marketing, v. 56, n.1, p. 6-21, 1992.

FORNELL et al. The American Customer Satisfaction Index: nature, purpose, and findings. Journal of Marketing, v. 60, n. 4, p. 7-18, 1996.

FORNELL, C.; LARCKER, D. F. Evaluating structural equation models with unobservable variables and measurement error. Journal of Marketing Research, $\mathrm{v}$. 18, n.1, p. 39-50, 1981.

GRÖNROOS, C. A service quality model and its marketing implications. European Journal of Marketing, v. 18, n. 4, p.36-44, 1984

HAIR et al. Partial least squares structural equation modeling (PLS-SEM): an emerging tool in business research. European Business Review, v. 26, n. 2, p.106$121,2014 a$.

HAIR et al. A Primer on Partial Least Squares Structural Equation Modeling (PLS-SEM). Los Angeles: SAGE, 2014b.

HENSELER, J.; RINGLE, C. M.; SINKOVICS, R. R. The use of partial least squares path modeling in international marketing. Advances in International Marketing, $\mathrm{v}$. 20, p. 277-319, 2009.

HENSELER, J.; HUBONA, G.; RAY, P. A. Using PLS path modeling in new technology research: updated guidelines. Industrial Management \& Data Systems, v. 116, n. 1 , p. $2-20,2016$.

IBGE (Instituto Brasileiro de Geografia e Estatística). 2017. Disponível em: <http://brasilemsintese.ibge.gov.br/servicos.html>. Acesso em 05 de junho de 2017. 


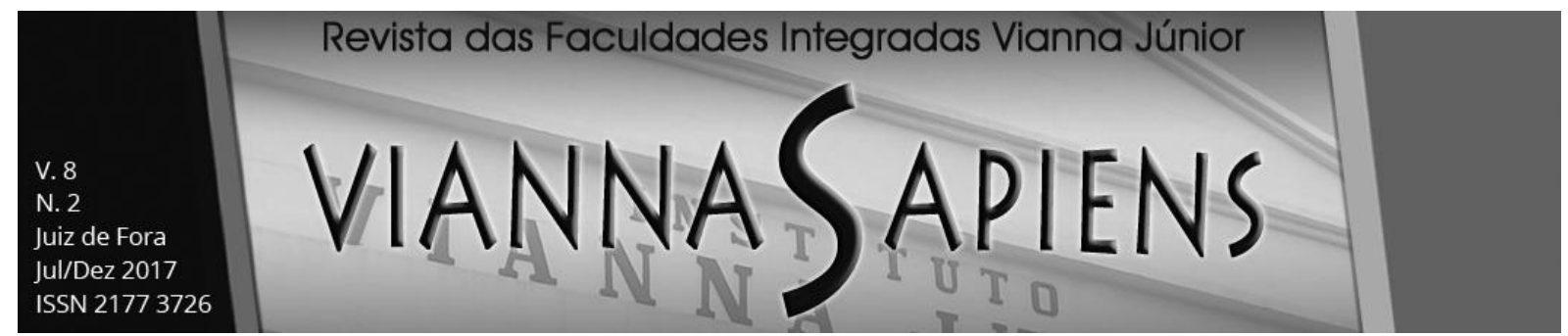

LOVELOCK, C.; WIRTZ, J.; HEMZO, M. A. Marketing de Serviços: pessoas, tecnologia e estratégia. 7 ed. São Paulo: Pearson Prentice Hall, 2011.

MARCHETTI, R.; PRADO, P. H. M. Um tour pelas medidas de satisfação do consumidor. RAE - Revista de Administração de Empresas, v. 41, n.4, p. 56-67, 2001.

OLIVER, R. L.A cognitive model of the antecedents and consequences of satisfaction decisions, Journal of Marketing Research, v. 17, n. 4, p. 460-469, 1980.

OLIVER, R. L. Whence consumer loyalty? The Journal of Marketing, v. 63, p. 3344, 1999.

OLIVER, R. L.; DESARBO, W. S. Response determinants in satisfaction judgments. Journal of Consumer Research, v. 14, n. 4, p. 495-507, 1988.

PARASURAMAN, A.; ZEITHAML, V. A.; BERRY, L. L. SERVQUAL: a multiple item scale for measuring consumer perceptions of service quality. Journal of Reatailing, v. 64 , n. 1, p. 12-40, 1988.

PARASURAMAN, A.; ZEITHAML V. A.; BERRY, L. L. Alternative scales for measuring service quality: a comparative assessment based on psychometric and diagnostic criteria. Journal of Retailing, v. 70, n. 3, p. 201-230, 1994.

RINGLE, C. M.; SILVA, D.; BIDO, D. Modelagem de equações estruturais com utilização do SmartPLS. Revista Brasileira de Marketing - ReMark, v.13, n.2, 2014. Edição especial.

SÁNCHEZ-GARCÍA et al. When satisfied consumers do not return: variety seeking's effect on short-and long-term intentions. Psychology \& Marketing, v. 29, n. 1, p. 1524, 2012.

SPRENG, R.; MACKENZIE, S.; OLSHAVSKY, R. A reeexamination of the determinants of consumer satisfaction. Journal of Marketing, v. 60, p. 15-32, 1996. 


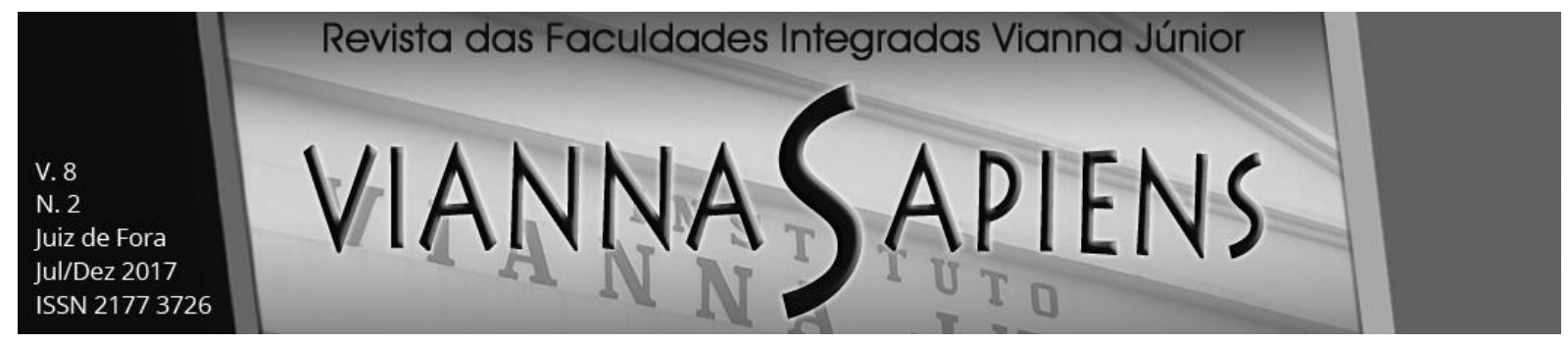

WOODRUFF, R. B. Customer Value: The Next Source for Competitive Advantage. Journal of the Academy of Marketing Science, v. 25, n. 2, p. 139-153, 1997.

YI, Y. The determinants of consumer satisfaction: the moderating role of ambiguity. Advances in Consumer Research, n. 20, p.502-506, 1993.

ZEITHAML, V. A. Consumer Perceptions of Price, Quality and Value: A Means-End Model and Synthesis of Evidence. Journal of Marketing, v, 52, n. 3, p. 2-22, 1988.

ZEITHAML, V. A.; BITNER, M. J.; GREMLER, D. D. Marketing de Serviços: A empresa com foco no cliente.Traduzido por Felix Nonnenmacher. 6 ed. Porto Alegre: Bookman, 2014. 